\title{
PANEL TESTING OF SPUTUM SMEAR MICROSCOPY OF NATIONAL TUBERCULOSIS REFERENCE LABORATORIES IN SAARC REGION: 2003-2010
}

\author{
Jha KK, Thapa B, Salhotra VS, Afridi NK \\ SAARC TB and HIVIAIDS Centre, Thimi, Nepal
}

\begin{abstract}
Introduction: Quality assured sputum smear microscopy is a priority for Tuberculosis Control Programme to be successful to meet the global TB targets of $70 \%$ case detection and $85 \%$ treatment success rate. The SAARC TB Reference Laboratory has been conducting Panel Testing under External Quality Assurance Sputum Smear Microscopy for National TB Reference Laboratories of SAARC Member States to strengthen the Sputum Smear Microscopy. The study aims to review and analyse the Panel Testing for National Reference Laboratories of SAARC Member States.
\end{abstract}

Methodology: The reports of seven rounds (2003-2010) of Panel Tests were collected and data from these reports was compiled and analysed.

Results: Seven rounds of Panel Testing in the region have been completed since 2003 and ten TB Reference Laboratories within eight Member States participated. Afghanistan has been participating since 2007. Seven hundred and seventy-eight panel test slides were dispatched to and read by the participating laboratories and percentage agreement was $98.07 \%$. Percentage agreement of individual participating laboratories varied from 87.50 to 100\%. Fourteen minor errors and one major error (high false positive) were also observed with sensitivity and specificity ranging from 90.90 to $100 \%$ and 83.33 to $100 \%$, respectively.

Conclusion: Panel Testing within the SAARC TB Laboratory network is extremely doing well. The success of TB control programmes in the SAARC Member States is reflected by the excellent Panel Testing within the SAARC TB Laboratory Network. Continuation and improvement of External Quality Assurance of Sputum Smear Microscopy, introduction of External Quality Assurance of culture \& drug susceptibility testing along with the introduction of recent diagnostic tests within the region is necessary to fight against TB.

Key words: Tuberculosis, Panel testing of sputum smear microscopy, SAARC TB reference laboratory network.

\section{INTRODUCTION}

TB is one of the major public health problems in the SAARC Region with immense socio-economic impacts. India, Bangladesh, Pakistan and Afghanistan are among 22 high-burden countries. ${ }^{1}$ Almost $50 \%$ the adult population of this Region

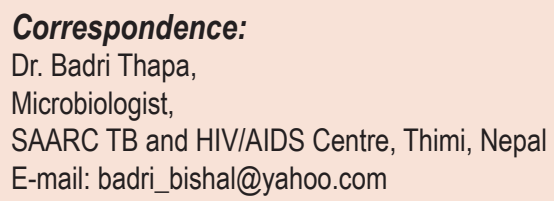

has already been infected with Mycobacterium tuberculosis and is at risk of developing tuberculosis disease. In the year 2009, a total of 2.0 million of all types of TB cases were notified in the SAARC region. This represents $74.07 \%$ of the 2.7 million estimated incident cases; the 0.8 million new smear positive cases notified account for 71.9 $\%$ of the 1.2 million estimates. The SAARC region carries $28.7 \%$ of the global burden of TB.

By adopting DOTS strategy, the Region has made remarkable progress in TB control. In the 
year 2008, SAARC Region covered $100 \%$ of its population under DOTS strategy. The Region has already achieved the target of $85 \%$ (now $88 \%$ ) treatment success rate and the target of $70.0 \%$ (now $71.9 \%$ ) case detection rate. ${ }^{1}$

The World Health Organization (WHO) Stop TB strategy for tuberculosis control (DOTS) relies on a network of laboratories that provide acid fast bacilli (AFB) microscopy. ${ }^{1}$ SAARC TB Reference Laboratory (STLR) and ten other National TB Reference Laboratories (NRLs), two each from India and Pakistan and one each from other Member States the SAARC TB Laboratory network. One of the objectives of SAARC TB and HIVIAIDS Centre (STAC) is strengthening laboratory network \& improving External Quality Assessment (EQA) and supervision. ${ }^{2}$

STRL at STAC is regularly supporting 10 National TB Reference Laboratories (NRLs) in 8 Member States in the area of quality assurance in sputum smear microscopy. Panel Testing-one of the three components for external quality assessment (EQA) or proficiency testing has been used to assure the quality of sputum smear microscopy services since $2003 .{ }^{3,4}$ So far, seven rounds of proficiency testing have been completed and eighth round is ongoing. This study aims to analyse seven rounds of proficiency tests for sputum smear microscopy within the network of National TB reference Laboratory of the SAARC region beginning from 2003 to 2010.

\section{METHODOLOGY}

The reports of seven rounds of proficiency tests were collected and data from these reports were compiled. ${ }^{2,5-10}$ The parameters that were studied were; number of Member States participated, number of slides included in each rounds, number of slides read and number of errors reported. The indicators of quality assessment of sputum smear microscopy were also calculated and the performance was analysed. The criteria of performance of the regional Panel Testing was interpreted as; $>95 \%=$ Excellent,
$90-95 \%=$ Satisfactory and $<90 \%=$ poor. Although, quantification error is considered as minor error in Panel Testing, it was taken as acceptable result for calculation of indicators in this analysis.

\section{RESULTS}

On behalf of STRL, National Tuberculosis Institute (NTI), Bangalore, India conducted the $1^{\text {st }}$ round of Panel Testing in 2003. NTI prepared the Panel Tests slides and were dispatched to seven NRLs including STRL which was also one of the participants. Since 2004 STRL is regularly conducting Panel Testing. Seventh round has been completed so far and eighth round is ongoing. Out of 10 NRLs in eight Member States that participated are currently participating in Panel testing, NTI and Tuberculosis Research Centre (TRC), Chennai, India participated since 2004 ( $2^{\text {nd }}$ round) and Afghanistan participated in 2007 ( $4^{\text {th }}$ round). The panel tests slides were prepared as per the guidelines "External Quality Assessment for AFB Smear Microscopy" and reported using "WHO/IUTALD" criteria for reporting AFB smear microscopy.

Number of panel slides received by all participating laboratories were 25 during the first round except for National Tuberculosis Reference Laboratory, Bangladesh, which received 23 slides and all laboratories are receiving 10 panel test slides from $2^{\text {nd }}$ round. Under panel Testing, out of the slides sent, five slides are stained and five are unstained. The positive and negative slides vary from round to round.

So far STRL has dispatched 778 panel test slides and correct results have been obtained for 763 slides (Table 1). The percentage agreement was $98.07 \%$. Out of 15 incorrect results, 8, 2, 1, and 4 represented low false negative, low false positive, high false positive and quantification error, respectively (Table 1). STLR received 25 panel test slides and there were no errors reported. Since its participation in 2007 (4th round), TB Reference Laboratory of Afghanistan has reported 40 slides with 5 incorrect results $(3,1$ and 1 are LFN, LFP and HFP results, respectively). 


\begin{tabular}{|c|c|c|c|c|c|}
\hline Country, Lab & $\begin{array}{c}\text { Year of } \\
\text { participation }\end{array}$ & $\begin{array}{l}\text { Participation } \\
\text { in round }\end{array}$ & $\begin{array}{l}\text { No. of } \\
\text { slides }\end{array}$ & $\begin{array}{c}\text { No. of } \\
\text { correct result }\end{array}$ & $\begin{array}{l}\text { No. of incorrect/acceptable } \\
\text { result (type) }\end{array}$ \\
\hline STRL & 2003 & 1 & 25 & 25 & - \\
\hline Afghanistan & $2007-2010$ & $4-7$ & 40 & 35 & 5 (3, LFN; 1, LFP; 1, HFP) \\
\hline Bangladesh & 2003-2010 & $1-7$ & 83 & 83 & - \\
\hline Bhutan & 2003-2010 & $1-7$ & 85 & 84 & $1(\mathrm{QE})$ \\
\hline India, NTI & $2004-2010$ & $2-7$ & 60 & 59 & $1(\mathrm{QE})$ \\
\hline India, TRC & $2004-2010$ & $2-7$ & 60 & 60 & - \\
\hline Maldives & 2003-2010 & $1-7$ & 85 & 82 & 3 (1, LFN; 2, QE) \\
\hline Nepal & 2003-2010 & $1-7$ & 85 & 85 & - \\
\hline Pakistan, OICD & 2003-2010 & $1-7$ & 85 & 83 & $2(1$, LFN; 2, LFP) \\
\hline Pakistan, FTC & 2003-2010 & $1-7$ & 85 & 84 & 1 (LFN) \\
\hline Sri Lanka & 2003-2010 & $1-7$ & 85 & 83 & 2 (LFN) \\
\hline Total & & & 778 & 763 & 15 \\
\hline
\end{tabular}

STRL, SAARC Tuberculosis Reference Laboratory; NTI, National Tuberculosis Institute; TRC, Tuberculosis Research Centre; OICD, Ojha Institute of Chest Disease; FTC, Federal Government Tuberculosis Centre; LFN, low false negative; LPF, low false positive; HFP, high false positive; QE, quantification error (acceptable result).

Public Health Laboratory of Bhutan has read 85 slides with only one incorrect result (Quantification Error) in the year 2008 (5th round). NTI, Bangalore and TRC, Chennai both from India participated since 2004 (2nd round). These laboratories have read 60 slides each and only one QE has been reported from NTI in the year 2009 (6th round). Indira Gandhi Memorial Hospital Laboratory of Maldives has been participating in Panel Testing since 2003 and 3 errors have been reported (1, low false negative; 2 Quantification Error). National TB Reference Laboratory, Nepal has not reported any errors since its participation in 2003. Ojha Institute of Chest Disease and Federal Government Tuberculosis Centre from Pakistan are participating since 2003 and 2 (1, LFN and 1, LFP) errors were reported, respectively. All of these errors were reported in 2010 (7th round). Like TB Reference Laboratories form Pakistan, TB Reference Laboratory of Sri Lanka reported one LFN result in 2010. No errors of any kind were reported by any laboratory in 2004 (2nd round).

The percentage agreement was more than $95 \%$ for 9 out of 10 participants and $100 \%$ agreement was seen for 5 laboratories (Table 2). Similarly, sensitivity was more $90 \%$ for all laboratories and specificity was more than $95 \%$ for all laboratories except for Afghanistan (83.33\%). 


\begin{tabular}{l} 
Table 2. Indicators of External Quality Assessment for Sputum Smear Microscopy in SAARC Laboratory \\
Network \\
\hline \multirow{4}{*}{ Country } \\
\hline
\end{tabular}

*QE are taken as acceptable result for calculating indicators; NRLs, National TB Reference Laboratories

\section{DISCUSSION}

WHO Stop TB strategy for tuberculosis control (DOTS) relies on a network of laboratories that provides acid fast bacilli (AFB) sputum smear microscopy. Sputum smear microscopy (SSM) is the diagnostic tool to diagnose TB at the field level in the resource limited settings. The generation time for Mycobacterium tuberculosis is 18-24 hours so the culture and identification needs $4-6$ weeks and further 4-6 weeks for drug susceptibility test. ${ }^{11}$ Molecular techniques are easy, rapid and of high sensitivity for TB diagnosis but they are not economical and difficult to adopt in resource limited countries. Hence, quality assured SSM is only the tool to bring success to TB control program in resource limited settings.

STLR and ten other NRLs of SAARC Member States are within the SAARC TB Laboratory network and the regional Panel Testing for SSM is being regularly conducted by STRL. Total agreement of the program in the region is $98.07 \%$. The success of TB control in the region is in part due to the quality assured SSM. By adopting DOTS strategy with a quality assured SSM, the
Region has achieved the global target of $70 \%$ of case detection (region, $71.9 \%$ ) and $85 \%$ treatment success (region, $88 \%$ ) and has made a remarkable progress in TB control. $^{1}$

The performance of Panel Testing was excellent (98.07\%). Performance of all the TB reference laboratories was more than $95 \%$ while TB Reference Laboratory of Afghanistan was less than $90 \%$. The Panel Testing conducted in Haiti showed $60 \%$ agreement and the sensitivity was below $80 \%$ for most of the laboratories assessed. However, the scoring criteria used in these two Panel testing were different. ${ }^{12}$ Similarly, the percentage agreement of $8^{\text {th }}$ round of Panel testing conducted at the district level in Bhutan was more than $90 \%$ for 15 out of 19 laboratories. ${ }^{13}$ The SAARC regional quality assurance for SSM has reported 778 panel slides, 15 were incorrect reports and most of them were low false negative $(n=8)$. Similarly, in $8^{\text {th }}$ Round of Panel testing conducted at the district level in Bhutan 27 errors were reported and 17 were low false. TB Reference Laboratories of Pakistan and Sri Lanka were doing well before 2010 in the Panel Testing conducted by STRL but few errors were reported in 2010. TB reference Laboratory of Maldives reported few errors $(n=3)$ in initial rounds $\left(1^{\text {st }}\right.$ and $\left.3^{\text {rd }}\right)$ of 
EQASSM but has performed well in recent rounds. More than 5000 bacilli/ml of sputum is necessary to yield positive SSM and errors are common if a person expectorates few bacilli in the sputum. ${ }^{14}$

Sensitivity and specificity ranged from 90.90 to $100 \%$ and 83.33 to $100 \%$, respectively. Errors in SSM are likely to result in failure to detect persons with active TB disease who can continue to spread the disease to the family members and to the community or unnecessary treatment for true negative cases. Errors in diagnosis can result in undue treatment of normal cases. Errors on follow up cases can result in overtreatment. Similarly, errors in detecting AFB on treatment failure cases can result in continued spread drug resistant TB in the community. Hence, error free SSM microscopy is utmost priority in the region. The high turnover of the staff and lack of training and refresher training affects the results. Hence, there should be training for new staff and refresher training for old staff on SSM. Case detection through quality assured SSM and their treatment through supply of quality assured drugs ensures the success of the TB control programme. But this also has a limitation that not all TB cases can be detected through SSM. Some TB patients who are sputum smear negative can also give TB culture positive as culture is more sensitive and can detect as low as 10 bacilli/ml of sputum..$^{15}$ Introduction of recent diagnostic test like, Gene Xpert MTB/Rif to detect M. tuberculosis would be helpful to fight against TB and achieve global targets.

\section{CONCLUSION}

The excellent EQASSM within the SAARC TB Laboratory Network is one of the contributors of the success behind the TB case detection, high treatment success rate and successful TB control programmes in the region. EQA for culture \& drug susceptibility testing is highly recommended within the STRL network to fight against TB.

\section{REFERENCES}

1. SAARC TB \& HIV/AIDS Centre, Tuberculosis in the SAARC Region, an update 2010.

2. SAARC TB \& HIV/AIDS Centre, Report of $6^{\text {th }}$ round proficiency testing of smear microscopy in National TB Reference Laboratories in SAARC region: 2009.
3. External Quality Assessment for AFB Smear Microscopy. APHL, CDC, KNCV, the Union, RIT, WHO. 2002.

4. Deun AV. External quality assessment of sputum smears microscopy: A matter of careful technique and organization. Int J Tuberc Lung Dis 2003;6:5078.

5. SAARC TB \& HIV/AIDS Centre, Report of $1 \mathrm{st}^{\mathrm{th}}$ round proficiency testing of smear microscopy in National TB Reference Laboratories in SAARC region: 2003.

6. SAARC TB \& HIV/AIDS Centre, Report of $2^{\text {nd }}$ round proficiency testing of smear microscopy in National TB Reference Laboratories in SAARC region: 2004.

7. SAARC TB \& HIV/AIDS Centre. Report of $3^{\text {rd }}$ round proficiency testing of Smear microscopy in National TB Reference Laboratories in SAARC region: 2005.

8. SAARC TB \& HIV/AIDS Centre. Report of $4^{\text {th }}$ round proficiency testing of smear microscopy in National TB Reference Laboratories in SAARC region: 2007.

9. SAARC TB \& HIV/AIDS Centre. Report of $5^{\text {th }}$ round proficiency testing of smear microscopy in National TB Reference Laboratories in SAARC region: 2008.

10. SAARC TB \& HIV/AIDS Centre. Report of $7^{\text {th }}$ round proficiency testing of smear microscopy in National TB Reference Laboratories in SAARC region: 2010.

11. Training manual for bacteriological aspects of drug resistance surveillance in India and SOP on culture and drug susceptibility testing procedures. The Central TB Division 2007. India

12. Baker $M$, Freeman $N$, Descombes $S$ et al. Implementation of external quality assessment (EQA) program for AFB smear microscopy in Haiti.

13. National Tuberculosis Reference Laboratory, National Public Health Laboratory, Bhutan. Report on AFB smear panel testing of district hospital laboratories. 2008.

14. Dunlap NE, Bass J, Fujiwara $P$ et al Diagnostic standards and classification of tuberculosis in adults and children. Am J Respir Crit Care Med 2000;161:1376-95

15. Long R. Smear negative pulmonary tuberculosis in industrialized countries. Chest 2001;120:330-34. 